\title{
EFECTOS DE LA MODULACIÓN DE FASE CRUZADA SOBRE LA PROPAGACIÓN DE ONDAS EN FIBRA ÓPTICA
}

\author{
Fideromo Saavedra G. ${ }^{1} \quad$ Álvaro Lamas N. ${ }^{2} \quad$ Marco Fernández B. ${ }^{2} \quad$ Yonatan Cepeda P. $^{2}$ \\ Recibido el 10 de agosto de 2005, aceptado el 26 de octubre de 2005
}

\begin{abstract}
RESUMEN
La modulación de fase cruzada (XPM) es un importante fenómeno que limita el desempeño de los sistemas ópticos de alta velocidad. En este trabajo se analizan y realizan tres modelos matemáticos diferentes que nos permiten comprobar y determinar las dependencias de XPM con respecto a la dispersión cromática de la fibra, la separación entre las longitudes de ondas, la potencia y la frecuencia de modulación.
\end{abstract}

Palabras clave: No linealidad, modulación de fase cruzada, propagación de pulsos.

\begin{abstract}
The cross-phase modulation (XPM) is an important phenomenon that limits the performance of the high speed optical systems. In this work three different mathematical models are analyzed and realiced, that they allow to verify and to determine the dependencies of XPM with respect to the chromatic dispersion of the fiber, the separation between the wavelengths, the power and the modulating frequency.
\end{abstract}

Keywords: Nonlinearity, cross-phase modulation, pulse propagation.

\section{INTRODUCCIÓN}

La transmisión de información a través de múltiples longitudes de ondas sobre la fibra óptica en sistemas WDM da origen a la aparición de efectos no lineales tales como la mezcla de cuatro ondas (FWM) y la modulación de fase cruzada (XPM) [1], [2]. El incremento en las tasas de transmisión hace que XPM sea un importante fenómeno que limita el desempeño de los sistemas ópticos, que utilizan fibra estándar, las que poseen una gran dispersión cromática en la región de los $1.55 \mu \mathrm{m}$ [3]-[5].

Los sistemas WDM/IM-DD no son sensibles a las fluctuaciones de la fase de la señal provocadas por XPM, por lo que éstas no son una fuente directa de la degradación [7]. No obstante, debido a la existencia de la dispersión de la fibra, las fluctuaciones de fase son convertidas en fluctuaciones de intensidad y éstas pueden degradar el desempeño del sistema [7], [8].
Para determinar el comportamiento de XPM en la propagación óptica a través de la fibra, se debe estudiar cómo afectan los distintos parámetros a la eficiencia de este fenómeno. En este trabajo se analizan, estudian y realizan tres modelos matemáticos diferentes que nos permiten comprobar y determinar las dependencias de XPM con respecto a la dispersión cromática de la fibra, la separación entre las longitudes de ondas, la potencia y la frecuencia de modulación. El primer modelo contempla el estudio de XPM a través de la resolución analítica y numérica de la ecuación no lineal de Schrödinger acoplada, observando los efectos temporales y espectrales de XPM sobre la propagación de múltiples pulsos. El segundo método consiste en la obtención, a partir de la ecuación de Schrödinger acoplada, de un índice de XPM que permite analizar las dependencias de este fenómeno. Por último el tercer modelo que, aislando el efecto de XPM en las ecuaciones de propagación, permite obtener una función de transferencia que comprueba y determina

\footnotetext{
1 Académico Departamento de Ingeniería Eléctrica, Universidad de Santiago de Chile, Casilla 10233, C. Central, Santiago, Chile, fsaavedr@lauca.usach.cl.

${ }^{2}$ Alumnos memoristas de Ingeniería Eléctrica, Universidad de Santiago de Chile, Santiago, Chile.
} 
el comportamiento de XPM bajo distintas condiciones de operación.

\section{MODELO PARA LA SIMULACIÓN DEL SISTEMA}

A continuación se hace una breve exposición de los tres modelos.

\section{A) Análisis de XPM mediante la solución de las ecuaciones de Schrödinger no lineales acopladas}

Una manera de analizar y poder aislar los efectos producidos por XPM es mediante una configuración de propagación de ondas, denominada de "bombeo y prueba" [5], [7] y [8], en la que una onda de "prueba" se introduce simultáneamente con una onda denominada de "bombeo", la que posee una mayor intensidad. Se produce así el ensanchamiento del espectro de prueba y se desarrollan oscilaciones en la amplitud. Sin embargo, a diferencia de SPM, XPM provoca un ensanchamiento asimétrico del canal de prueba, debido a que el cambio de fase no lineal incluye el desequilibrio de las velocidades de grupo y las diferencias de potencia.

Cuando se tienen dos ondas propagándose en una fibra óptica monomodo, el término de acoplamiento de XPM de los pulsos puede afectar la forma y el espectro de ambos pulsos. En este sentido la dispersión de la velocidad de grupo juega un papel importante, afectando a XPM.

En primer lugar, es responsable de un desequilibrio entre las velocidades de grupo asociadas a los pulsos. Como resultado, cada pulso pasa a través del otro mientras ellos se propagan a lo largo de la fibra. La interacción de XPM deja de ocurrir cuando los pulsos se separan [8], [9].

Un parámetro útil en el estudio de este fenómeno, es la longitud de “Walk off', que está dada por [9]:

$$
L_{W}=\frac{T_{0}}{\left|d_{12}\right|}
$$

con $d_{12}$ parámetro de "Walk off" que entrega información sobre la diferencia entre las velocidades de grupo. La longitud de "Walk off" permite estimar cuanto durará la interacción entre los pulsos. Así XPM ocurrirá a distancias aproximadamente iguales a $L_{w}$ de la longitud de la fibra.

Otro efecto que tiene la dispersión es producido a través de los términos correspondientes a las dispersiones de la velocidad de grupo (GVD). La importancia de los efectos GVD puede ser estimada mediante la longitud de dispersión, definida por [9]:

$$
L_{D}=T_{0}^{2} /\left|\beta_{12}\right|
$$

El estudio de la propagación de pulsos en la fibra parte con la ecuación de Schrödinger acoplada que está dada por [10], [11]:

$$
\begin{aligned}
\frac{\partial u_{i}(z, t)}{\partial z}+ & \beta_{i 1} \frac{\partial u_{i}(z, t)}{\partial t}-j \frac{\beta_{i 2}}{2} \frac{\partial^{2} u_{i}(z, t)}{\partial t^{2}}+\frac{\alpha}{2} u_{i}(z, t) \\
& +j \gamma_{i} \cdot\left(P_{0 i}\left|u_{i}(z, t)\right|^{2}+2 P_{0 m} \sum_{m \neq i}^{M}\left|u_{m}(z, t)\right|^{2}\right) u_{i}(z, t)=0
\end{aligned}
$$

donde el módulo al cuadrado de la variable $u_{1}(z, t)$ representa la potencia instantánea normalizada a su valor máximo $P_{01}$ de la envolvente lenta $A_{i}(z, t)$ de la i- ésima longitud de onda, $\beta_{i 1}$ y $\beta_{i 2}$ se relacionan con la velocidad de grupo y la dispersión de la velocidad de grupo respectivamente, para la i-ésima longitud de onda. El parámetro $a$ representa el coeficiente de pérdidas de la fibra y se ha supuesto independiente de la longitud de onda. El parámetro $\gamma_{i}$ es el coeficiente de no linealidad de la fibra cuya expresión es:

$$
\gamma_{i}=\frac{n_{2}}{A_{e f f}} \frac{\omega_{0 i}}{c}
$$

con $c$ velocidad de la luz en el vacío y $A_{e f f}$ área efectiva sobre la cual está distribuida la potencia del modo propagado, asumiendo que es casi idéntica para cada uno de los canales y $\mathrm{n}_{2}$ representa la constante de proporcionalidad no lineal o índice Kerr, producto de la dependencia del índice de refracción con la intensidad del campo eléctrico de la onda. Los términos que acompañan al coeficiente de no-linealidad representan la contribución del fenómeno de automodulación de fase y modulación de fase cruzada, respectivamente.

Considerando, además, un sistema de dos señales ópticas, señales de prueba y bombeo, donde la longitud de dispersión es mucho más grande que $L$ y $L_{W}$, los términos de dispersión de la velocidad de grupo en la ecuación (3) pueden ser despreciados, por lo que las formas temporales de los pulsos no se verán afectadas, debido a que no hay variación en el tiempo de la envolvente lenta. Despreciando también las perdidas de la fibra, debido a que para este análisis las longitudes son tales que $L \approx L_{W}$, podemos expresar la ecuación (3) como:

$\frac{\partial u_{i}(z, t)}{\partial z}+\frac{1}{v_{g i}} \frac{\partial u_{i}(z, t)}{\partial t}+j \gamma_{i} \cdot\left(P_{0 i}\left|u_{i}(z, t)\right|^{2}+2 P_{0 k}\left|u_{k}(z, t)\right|^{2}\right) u_{i}(z, t)=0$

con $\mathrm{i}, \mathrm{k}=1,2$ pero no iguales. Esta expresión tiene una solución general del tipo [5]: 


$$
u_{i}(z, t)=u_{i}(0, t-z / v g i) \exp \left(j \phi_{i}(z, t)\right)
$$

Ésta permite determinar como la envolvente del pulso a lo largo de la propagación es afectada espectralmente. Si separamos la variable compleja de la ecuación (5) se tendrá:

$$
\frac{\partial \phi_{i}(z, t)}{\partial z}=-\gamma_{i}\left(P_{o i}\left|u_{i}\left(0, t-z / v_{g i}\right)\right|^{2}+2 P_{0 k}\left|u_{k}\left(0, t-z / v_{g k}\right)\right|^{2}\right)
$$

Esta expresión tiene una solución en $z=L$ de la forma:

$$
\begin{aligned}
\phi_{i}(z, t)=-\gamma_{i}\left(P_{o i} L\left|u_{i}\left(0, t-L / v_{g i}\right)\right|^{2}\right. \\
\left.+2 P_{0 k} \int_{0}^{L}\left|u_{k}\left(0, t-L / v_{g k}+d_{i k} z^{\prime}\right)\right|^{2} d z^{\prime}\right)
\end{aligned}
$$

con lo que se sabe cómo se comporta la fase del pulso para un instante determinado que, en este caso, se ha supuesto para $z=L$. Sin embargo, es necesario determinar la forma de los pulsos de entrada $u_{i}(0, t-z / v g i)$. Definiendo, por simplicidad, un pulso gaussiano y sin chirp inicial, se tendrá para ambos pulsos que:

$$
u_{1}(0, t)=u_{2}(0, t)=\exp ^{-\frac{t^{2}}{2}}
$$

Así se define, completamente y de manera analítica, cómo evoluciona el pulso espectralmente a lo largo de la fibra bajo los efectos de SPM (automodulación de fase) y XPM.

Si $L$ viene a ser comparable a $L_{D}$, los efectos combinados de XPM y GVD conducen a nuevos cambios temporales, que acompañan los cambios espectrales, por lo que la expresión analítica no nos sirve para describir la evolución temporal y espectral de los pulsos a lo largo de la fibra y es necesario resolver numéricamente la ecuación (3) [8]. En este trabajo se emplea para la resolución de la ecuación de propagación, en una fibra monomodo estándar, el método de Fourier de paso dividido simetrizado [8], [10].

\section{B) Estudio de XPM mediante el índice de eficiencia en enlaces de Fibra Óptica}

El análisis de XPM anterior está relacionado con la propagación de pulsos en fibra óptica. Esto trae como consecuencia que, en el caso de que la dispersión, juegue un papel importante; será necesario emplear métodos numéricos para poder resolver estas ecuaciones. No obstante es posible obtener un parámetro de eficiencia de XPM, que muestra la incidencia que tienen los parámetros del sistema sin necesidad de resolver las ecuaciones [6].
En esta sección se analizará el fenómeno de XPM en un sistema óptico que consta de dos canales, en una configuración de prueba y bombeo, para un segmento de fibra óptica. Los resultados de este análisis dan origen a un modelo matemático que describe la modulación de fase cruzada en enlaces ópticos de este tipo, modelo que será simulado. La Fig. 1 muestra un esquema del sistema:

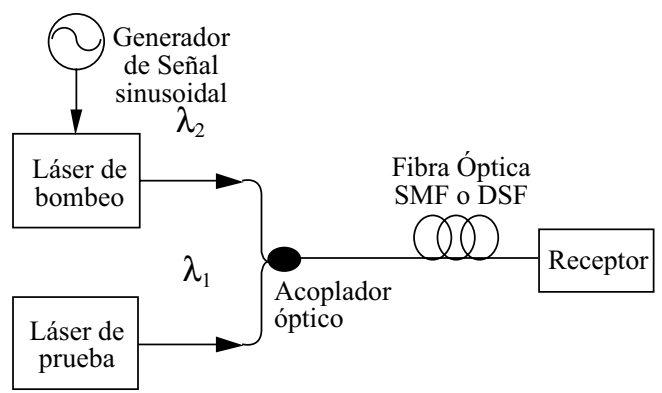

Fig. 1 Enlace de fibra óptica de un segmento.

\section{B.1) Modulación Sinusoidal}

Consideremos dos pulsos ópticos con la misma polarización propagándose en una fibra óptica monomodo. Se normaliza la envolvente del campo de cada onda $A_{i}(z, t)$ y $|A|^{2}$ igual a la potencia óptica instantánea como puntos de partida. Si el parámetro GVD no produce cambios apreciables en la forma de $A_{i}(z, t)$, pero hace que las ondas se propaguen a diferentes velocidades de grupo, podemos depreciar los parámetros de GVD y expresar (3) como [5], [6]:

$$
\frac{\partial A_{i}}{\partial z}+\frac{\alpha}{2} A_{i}+\frac{1}{v_{g i}} \frac{\partial A_{i}}{\partial t}=j \gamma_{i}\left(\left|A_{i}\right|^{2}+2\left|A_{k}\right|^{2}\right) A_{i}
$$

Donde $i, k=1$ ó $2, \mathrm{i} \neq \mathrm{k}$ y $v_{g i}$ es la velocidad de grupo de la onda $i$. Los dos términos en el lado derecho de la ecuación (10) son consecuencia del índice de refracción no lineal. La solución general de la Ec. (10) tendrá la forma [5]:

$$
A_{i}(z, t)=A_{i}\left(0, t-z / v_{g i}\right) \exp (-\alpha z / 2) \exp \left[j \phi_{i}(z, t)\right]
$$

Donde:

$$
\phi_{i}(z, t)=\gamma_{i}\left[\frac{1-e^{-\alpha z}}{\alpha}\left|A_{i}\left(0, t-z / v_{g i}\right)\right|^{2}+2 \int_{0}^{z}\left|A_{k}\left(0, t-z / v_{g i}+d_{i k^{\prime}} z^{\prime}\right)\right|^{2} e^{-\alpha z^{\prime}} d z^{\prime}\right]
$$

es el cambio de fase inducido por SPM y XPM, respectivamente. El parámetro de "walk-off" $d_{i k}$ se define como [9]: 


$$
d_{i k}=\left(v_{g i}\right)^{-1}-\left(v_{g k}\right)^{-1}=\int_{\lambda_{k}}^{\lambda_{i}} D(\lambda) d \lambda
$$

En una región con dispersión distinta de cero, el parámetro de "walk-off" se aproxima a [5]: $d_{i k}=D \cdot \lambda_{i k}$, donde D es el coeficiente de la dispersión y $\lambda_{\mathrm{ik}}=\lambda_{\mathrm{i}}-\lambda_{\mathrm{k}}$ es la separación en longitud de onda entre los dos canales.

Si el canal 1 (de prueba) es CW (señal continua) y la intensidad del canal 2 (bombeo) se modula de manera sinusoidal con una frecuencia angular $\omega$, las expresiones para la potencia óptica de ambos canales en $\mathrm{z}=0$ (principio de la fibra) están dadas por:

$$
\begin{gathered}
P_{1}(0, t)=\left|A_{1}(0, t)\right|^{2}=P_{10} \\
P_{20}=\left|A_{2}(0, t)\right|^{2}=P_{20}+P_{2 m} \cos (\omega t+\theta)
\end{gathered}
$$

Donde $P_{2 m}$ es la amplitud de la potencia de la modulación sinusoidal y $\theta$ corresponde a una fase inicial arbitraria. Sustituyendo la Ec. (14) y la Ec. (15) en la Ec. (12) se obtiene la fase de la portadora óptica [5]:

$$
\phi_{1}(L, t)=\gamma_{1}\left(P_{10}+2 P_{20}\right) L_{e f f}+\Delta \phi_{1} \cos \left[\omega\left(t-L / v_{g 1}\right)+\theta+\varphi\right](16)
$$

aquí L es el largo de la fibra; Leff $=\left(1-e^{-\alpha L}\right) / \alpha$ es el largo efectivo de la fibra; $\Delta \phi_{1}$ es la amplitud del cambio de fase inducido por la XPM; $\theta$ es el factor de retardo de fase de XPM asociado con $\omega ; \phi$ es la diferencia de fase entre la potencia óptica modulada de manera sinusoidal $\mathrm{P}_{2}$ y la potencia $\mathrm{P}_{1}$ del canal de prueba.

De la ecuación (16) se encuentra que $\Delta \phi_{1}$ es proporcional a $P_{2 m}$ y está dado por [5]:

$$
\Delta \phi_{1}=2 \gamma_{1} P_{2 m} \sqrt{\eta_{X P M}} L_{e f f}
$$

Donde $\eta_{X P M}$ se define como la eficiencia del fenómeno XPM y está dado por [5]:

$$
\eta_{X P M}=\frac{\alpha^{2}}{\omega^{2} d_{12}^{2}+\alpha^{2}}\left[1+\frac{4 \operatorname{sen}^{2}\left(\frac{\omega d_{12} L}{2}\right) e^{-\alpha L}}{\left(1-e^{-\alpha L}\right)^{2}}\right]
$$

La ecuación (17) puede ser normalizada escribiéndola de la forma $\Delta \phi_{1} / P_{2 m}=2 \gamma_{1} \sqrt{\eta_{X P M}} L_{e f f}$, expresión que será simulada para enlaces ópticos de un segmento.

Las ecuaciones (16) y (18) muestran que la fase de la señal de prueba está modulada en $\omega$. La intensidad de esta modulación de fase depende de los parámetros $\gamma_{1}$, $\mathrm{P}_{2 \mathrm{~m}}, \mathrm{~L}_{\text {eff }} \mathrm{y} \eta_{\mathrm{XPM}}$. Por otra parte, el parámetro $\eta_{\mathrm{XPM}}$ dado por la ecuación (18) depende de $\mathrm{D}, \lambda$ y $\omega$.

\section{C) Función de transferencia normalizada para la potencia de XPM}

Los modelos matemáticos para el cálculo de XPM se obtienen del análisis de la ecuación de Schrödinger acoplada [9], dada por la ecuación (3). En este tercer modelo se consideran dos señales ópticas, de prueba $\mathrm{y}$ bombeo, $A_{j}(t, z)$ y $A_{k}(t, z)$ respectivamente, propagándose en la fibra óptica. Los parámetros $\lambda_{j} \mathrm{y} \lambda_{k}$ son la longitud de onda de la señal de prueba y bombeo, respectivamente. Se definen para este análisis $p_{k}=\left|A_{k}\right|^{2} \mathrm{y} p_{j}=\left|A_{j}\right|^{2}$ como las potencias ópticas de la señal de bombeo y prueba, respectivamente.

Debido a la dispersión cromática, las ondas de prueba y bombeo poseen diferentes velocidades de grupo y esta diferencia debe tenerse en cuenta en el cálculo de XPM. También se utiliza el parámetro “Walk-off' entre las dos ondas realizándose una aproximación lineal, donde el "Walk-off" es expresado como $d_{j k}=S_{o}\left(\lambda-\lambda_{o}\right) \cdot \Delta \lambda_{j k}$, con $\lambda_{\mathrm{o}}$ como la longitud de onda de dispersión cero de la fibra, $\mathrm{S}_{\mathrm{o}}$ es la pendiente de la dispersión y $\Delta \lambda_{j k}$ es el espaciamiento de longitud de onda entre las señales de prueba y bombeo. La señal de prueba opera en modo de onda continua $(\mathrm{CW})$ y la señal de bombeo es modulada con una onda senoidal a una frecuencia $\Omega$. Con el objetivo de enfocar el análisis en el estudio de XPM, se obvia el efecto de SPM en ambos canales. Así eliminamos la primera variable del quinto término en el lado izquierdo de la Ec.(3). Usando la substitución $T=t-z / v_{j} \mathrm{y}$ $A_{j}(t, z)=E_{j}(T, z) \exp (-\alpha z / 2)$, se tiene [7]:

$$
\begin{aligned}
& \frac{\partial E_{j}(T, z)}{\partial z}=-i \frac{\beta_{2}}{2} \cdot \frac{\partial^{2} E_{j}(T, z)}{\partial T^{2}}+ \\
& +i \gamma_{j} 2 p_{k}\left(T-d_{j k} z, 0\right) \cdot \exp (-\alpha z) E_{j}(T, z)
\end{aligned}
$$

En general, la dispersión y la no linealidad actúan juntos a lo largo de la fibra. Sin embargo, en una sección dz infinitesimal de fibra, podemos asumir que la dispersión y los efectos no lineales actúan independientemente.

Tomando en cuenta solamente el efecto de modulación de fase cruzada, con $\mathrm{z}=\mathrm{z}$ ', la modulación de fase no lineal en la señal de prueba, inducida por la señal de bombeo en la pequeña sección $d z$, puede obtenerse como:

$$
d \phi_{j}\left(T, z^{\prime}\right)=2 \gamma_{j} p_{k}\left(T-d_{j k} z^{\prime}, 0\right) \exp (-\alpha z) d z
$$

La transformada de Fourier de la variación de fase es:

$$
d \tilde{\phi}_{j}\left(\Omega, z^{\prime}\right)=2 \gamma_{j} p_{k}(\Omega, 0) \exp \left(-\alpha+i \Omega d_{j k}\right) z^{\prime} d z
$$


Debido a la dispersión cromática de la fibra, esta variación de fase, generada con $z=z$ ', es convertida en una variación de amplitud en el largo de la fibra $z=L$. Teniendo en cuenta sólo la dispersión y una fuente terminal de la perturbación de fase con $\mathrm{z}=\mathrm{z}$, la transformada de Fourier de (19) es:

$$
\frac{\partial \tilde{E}_{j}(\Omega, z)}{\partial z}=i \frac{\beta_{2} \Omega^{2}}{2} \cdot \tilde{E}_{j}(\Omega, z)+\bar{E}_{j}\left[1+i d \tilde{\phi}\left(\Omega, z^{\prime}\right)\right] \delta\left(z-z^{\prime}\right)
$$

donde $\delta\left(z, z^{\prime}\right)$ toma en cuenta que la fuente terminal existe sólo en una sección infinitesimal de la fibra $z=z^{\prime}$ [7]. Por lo tanto, en la salida de la fibra, a $\mathrm{z}=\mathrm{L}$, el campo de la señal de prueba es:

$$
\tilde{E}_{j}(\Omega, L)=\bar{E}_{j}+i d \tilde{\phi}_{j}\left(\Omega, z^{\prime}\right) \bar{E}_{j} \exp \left[\frac{i \beta_{2} \Omega^{2}\left(L-z^{\prime}\right)}{2}\right](23)
$$

La variación de la potencia óptica causada por la no linealidad de la modulación de fase, creada en la sección $d z \operatorname{con} \mathrm{z}=\mathrm{z}$, es:

$$
\begin{aligned}
& \Delta \tilde{a}_{j k}\left(\Omega, z^{\prime}, L\right)=\left|\tilde{E}_{j}(\Omega, L)\right|^{2}-\bar{E}_{j}^{2}= \\
& \quad=-2 \bar{E}_{j}^{2} d \tilde{\phi}_{j}\left(\Omega, z^{\prime}\right) \sin \left[\beta_{2} \Omega^{2} \frac{\left(L-z^{\prime}\right)}{2}\right]
\end{aligned}
$$

donde se hace una linealización, considerando que $d \tilde{\phi}_{j}$ es infinitesimal. Usando:

$E_{j}(T, z)=A_{j}\left(T+z / v_{,} z\right) \exp (\alpha z / 2),(21)$ e integrando toda la modulación de fase cruzada a lo largo de la fibra, obtenemos la fluctuación de intensidad total al final de la fibra:

$$
\begin{aligned}
& \Delta \tilde{S}_{j k}(\Omega, L)=2 p_{j}(L) \gamma_{j} \exp \left(i \Omega / v_{j} L\right) p_{k}(\Omega, 0) \\
& \left\{\frac{\exp \left(i \beta_{2} \Omega^{2} L / 2\right)-\exp \left(-\alpha+i \Omega d_{j k}\right) L}{i\left(\alpha-i \Omega d_{j k}+i \beta_{2} \Omega^{2} / 2\right)}-\frac{\exp \left(i \beta_{2} \Omega^{2} L / 2\right)-\exp \left(-\alpha+i \Omega d_{j k}\right) L}{i\left(\alpha-i \Omega d_{j k}-i \beta_{2} \Omega^{2} / 2\right)}\right\}
\end{aligned}
$$

Asumiendo que $\exp (-\alpha L)<<1$ y que el ancho de banda de la modulación es más pequeño que el espaciamiento de canal, encontramos en el dominio de la frecuencia, una descripción más simple de la fluctuación de intensidad en el canal de prueba causada por la modulación de intensidad del canal de bombeo:

$$
\Delta \tilde{S}_{j k}(\Omega, L)=4 \gamma_{j} p_{j}(L) p_{k}(\Omega, 0) \frac{\sin \left(\frac{\beta_{2} \Omega^{2} L}{2}\right)}{\alpha-i \Omega d_{j k}} \exp \left(\frac{i \Omega L}{v_{j}}\right)(26)
$$

Esta ecuación se emplea en el análisis de la respuesta en frecuencia de XPM.

\section{RESULTADOS}

A) Mediante la solución analítica del pulso obtenida con las expresiones (6) y (8), podemos representar el espectro de dos pulsos en una configuración de bombeo y prueba. Considerando un sistema con retardo inicial cero y usando un pulso en $630 \mathrm{~nm}$, con $100 \mathrm{~mW}$ de potencia peak, junto con otro pulso en $530 \mathrm{~nm}$, con $50 \mathrm{~mW}$ de potencia peak, y los valores $\mathrm{T}_{0}=10 \mathrm{ps}, L=5 \mathrm{~m}, \gamma_{1} P_{01} L=40, P_{02} / P_{01}=0.5$ y $\gamma_{2} / \gamma_{1}=1.2$, con $\delta=\frac{d L}{T_{0}} \quad$ [8], se obtienen los espectros ópticos de ambos pulsos mostrados Fig. 2.
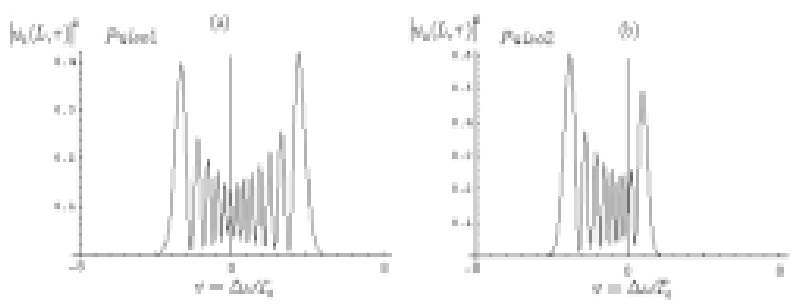

Fig. 2 Espectro óptico para la intensidad de los pulsos (a) pulso 1 (bombeo) y (b) pulso 2 (prueba), después de propagarse $\mathrm{z}=\mathrm{L}$ y $\mathrm{T}_{0}=10 \mathrm{ps}$.

Las figuras 2(a) y 2(b) muestran la asimetría del ensanchamiento espectral de ambos pulsos, producida por XPM. Nótese que el cambio espectral del pulso 2 es mayor, debido a que la contribución de XPM es más grande para este pulso, por haber considerado $\mathrm{P}_{1}>\mathrm{P}_{2}$. Cuando $\mathrm{P}_{1}=\mathrm{P}_{2}$, y no se considera la presencia de XPM, los cálculos demuestran que los ensanchamientos de los dos espectros son simétricos y menores.

Si $L$ viene a ser comparable a $L_{D}$, los efectos combinados de XPM y GVD conducen a nuevos cambios temporales, que acompañan los cambios espectrales. En este caso los términos correspondientes a la dispersión de la velocidad de grupo no pueden ser despreciados $\mathrm{y}$, por lo tanto, la ecuación (3) no puede ser resuelta analíticamente y se hace necesaria una aproximación numérica. Así, empleando el método de Fourier de paso dividido simetrizado y considerando un caso, en que: $L_{D} / L_{W}=10$, $\omega_{2} / \omega_{1}=1.2$ y $10^{2}=\frac{\gamma_{1} P_{1} T_{0}^{2}}{\left|\beta_{1}\right|}, L_{0}=6000 \mathrm{~m}[8]$, con $\mathrm{P} 1>>\mathrm{P} 2$, con ambos pulsos propagándose en régimen de dispersión normal y que tanto el pulso de prueba como el de muestreo son pulsos gaussianos de igual ancho y sin retardo inicial, se obtienen las siguientes representaciones temporales: 


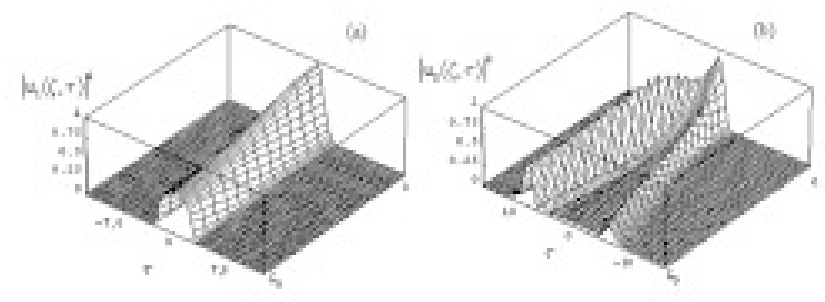

Fig. 3 Evolución temporal de los pulsos ópticos de(a) bombeo y b) prueba en presencia de dispersión GVD y no linealidad, para $\mathrm{L}=6.000 \mathrm{~m}$.

Una característica que diferencia XPM de SPM es que la forma del ensanchamiento del pulso es asimétrica con sólo un lado desarrollando oscilaciones. En el caso de la Fig. 3 estas oscilaciones se presentan en el lado derecho, debido a que el pulso de bombeo interactúa con ese lado del pulso. Se espera que la evolución de los espectros de ambos pulsos, también presenten variaciones, tal como se muestra en la Fig. 4:
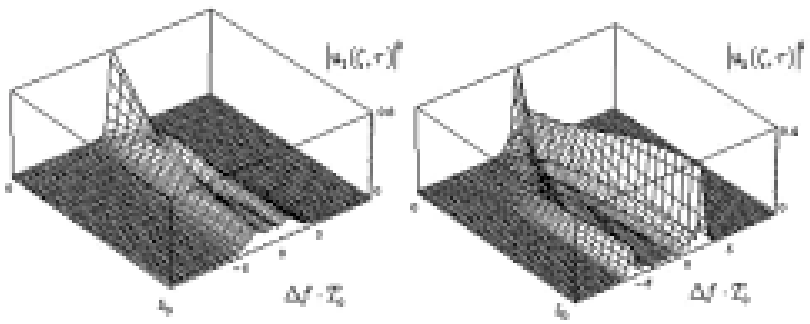

Fig. 4 Evolución espectral de los pulsos ópticos de (a) bombeo y (b) prueba, en presencia de dispersión GVD y no linealidad, para $\mathrm{L}=6.000 \mathrm{~m}$.

Como se observa en la Fig. 4(a) el pulso de bombeo presenta débiles cambios en su estructura debido a que la intensidad del pulso de prueba es muy pequeña comparada a la intensidad del pulso de bombeo, por lo que el espectro es simétrico. Sin embargo el espectro del pulso de prueba presenta un ensanchamiento asimétrico y de naturaleza oscilatoria, efecto producido por XPM.

B) Se simula un enlace óptico de un segmento como el de la Fig. 1. Se genera un gráfico de la Ec. (17) normalizada, denominada índice de XPM normalizado en función de la frecuencia de modulación de la señal de bombeo y de la separación entre las longitudes de onda de la señal de prueba y de bombeo.

La fibra óptica empleada en la simulación computacional es del tipo monomodo estándar con los parámetros dados por [12]. El valor del coeficiente del índice de refracción no lineal de la fibra óptica es $\mathrm{n}_{2}=2,35 \times 10^{-20} \mathrm{~m}^{2} / \mathrm{W}$. El diámetro del campo modal (w) es de $10,5 \mu \mathrm{m}$ y la atenuación de esta fibra es de $0,25 \mathrm{db} / \mathrm{km}$ a $1550 \mathrm{~nm}$. El parámetro de dispersión cromática $\mathrm{D}$ tiene un valor de $17 \mathrm{ps} / \mathrm{km} \cdot \mathrm{nm}$. La longitud de onda de la señal de prueba $\lambda_{1}$ se fija en $1559 \mathrm{~nm}$ y la longitud de onda de la señal de bombeo $\lambda_{2}$ se fija en $1559,2 \mathrm{~nm}$. La longitud de onda de operación del sistema es de $1559,1 \mathrm{~nm}$ y el largo del enlace es de $50 \mathrm{~km}$.

La Fig. 5 muestra un gráfico del índice de XPM normalizado versus la frecuencia de modulación de la señal de bombeo para un enlace óptico como el de la Fig. 1.

Se observa que el índice de XPM es mayor cuando se disminuye la separación entre las longitudes de onda y que depende fuertemente de este parámetro.

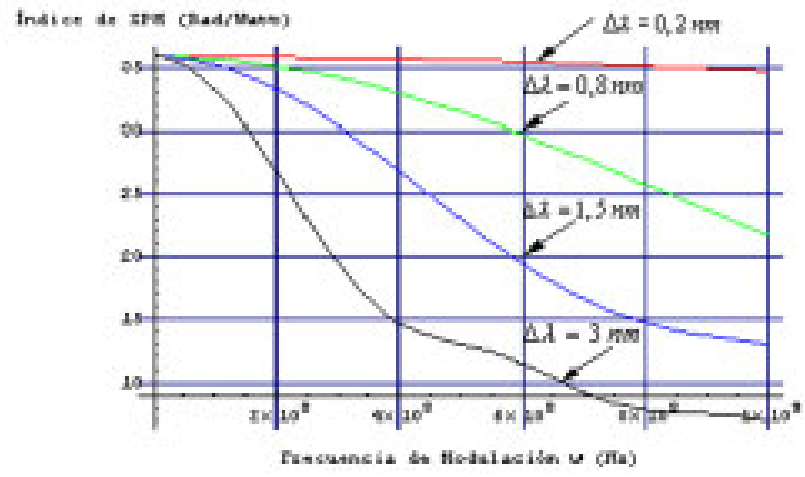

Fig. 5 Índice de XPM versus frecuencia de modulación de la señal de bombeo.

C) Por último presentamos los resultados obtenidos para el cálculo de la respuesta en frecuencia de la diafonía inducida por XPM para una configuración de prueba y bombeo sobre un enlace de fibra estándar monomodo de un segmento, análoga a la mostrada en la Fig. 1. En este análisis los parámetros de la fibra son: $\mathrm{L}=50 \mathrm{Km}$, $\lambda_{\mathrm{o}}=1550 \mathrm{~nm}, \mathrm{D}=17 \mathrm{Ps} / \mathrm{Km} \cdot \mathrm{nm}, \alpha=0.25 \mathrm{~dB} / \mathrm{Km}$, $\mathrm{n}_{2}=2.35 \times 10^{-20} \mathrm{~m}^{2} / \mathrm{W}, \lambda_{\mathrm{j}}=1559 \mathrm{~nm}, \mathrm{~A}_{\text {eff }}=8.7 \times 10^{-11} \mathrm{~m}^{2}$, Frecuencia de Modulación (bombeo) $50 \mathrm{MHza} 10 \mathrm{GHz}$ [12].

La Fig. 6 muestra la respuesta en frecuencia de la diafonía inducida por XPM, para distintas potencias de la señal de bombeo, con una separación entre las longitudes de onda de $\Delta \lambda j=0.2 \mathrm{~nm}$.

Se observa que la respuesta en frecuencia de XPM es fuertemente dependiente de la potencia de la señal de bombeo. Esto se debe a que el término de fase de la señal de prueba depende de la potencia del canal vecino, que para este caso es la señal de bombeo.

Si se aumenta la separación entre las longitudes de ondas para los distintos niveles de potencia de la señal de bombeo, se comprueba, con el mismo método, que la respuesta en frecuencia de XPM disminuye aproximadamente $5(\mathrm{~dB})$ para cada uno de los casos. 


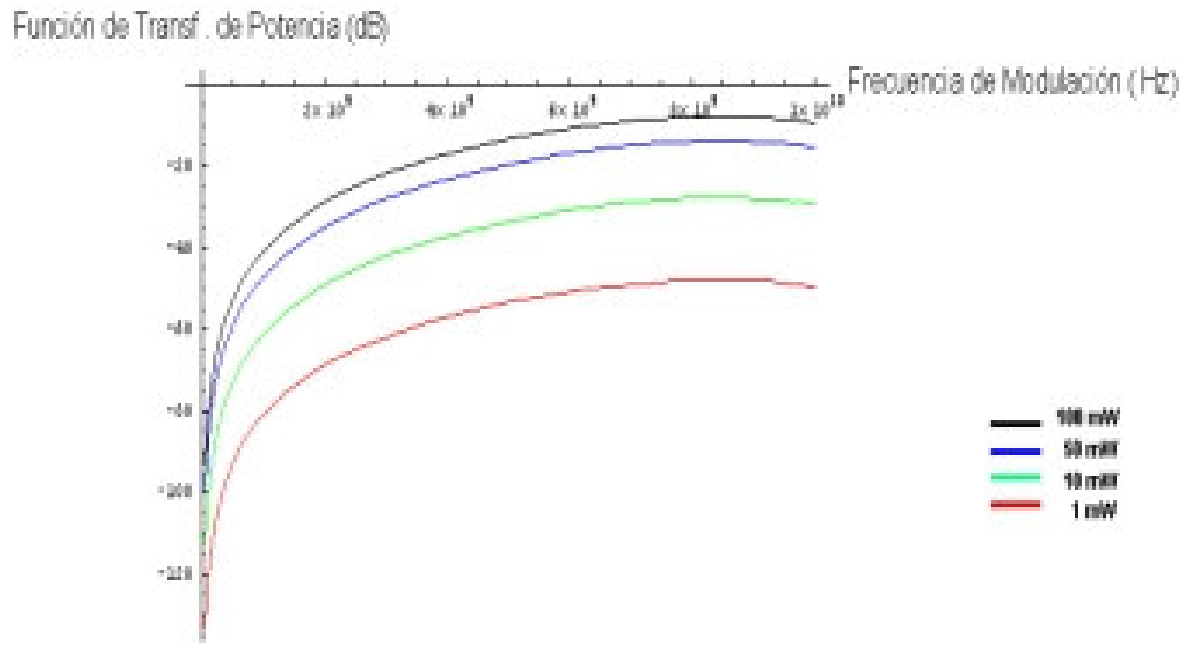

Fig. 6 Respuesta en frecuencia de la diafonía inducida por XPM, para distintas potencias $\operatorname{conDl}_{\mathrm{jk}}=0.2 \mathrm{~nm}$.

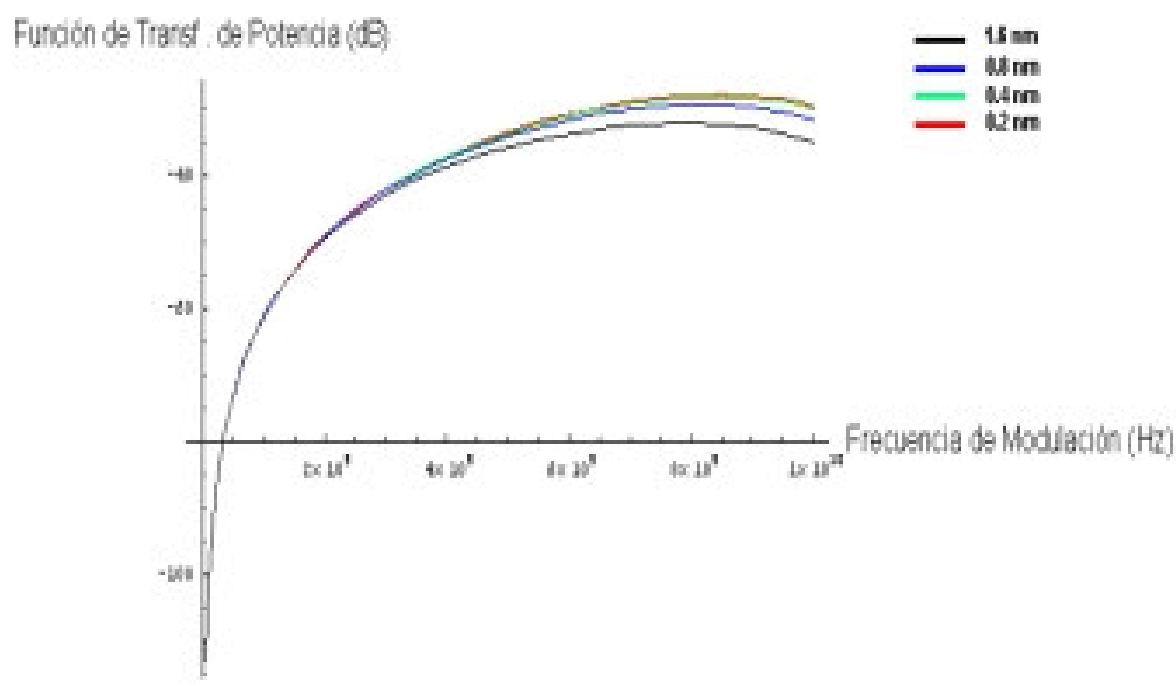

Fig. 7 Respuesta en frecuencia de la diafonía inducida por XPM, para diferentes separaciones entre las longitudes de onda con potencia $=10 \mathrm{~mW}$.

La dependencia de XPM con la separación entre los canales, se puede observar mejor en un gráfico de la respuesta en frecuencia de XPM, considerando distintas separaciones entre canales, como se observa en la Fig. 7. Allí se muestran las respuestas en frecuencia de tipo filtros pasa alto. Debe señalarse que este modelo muestra características cualitativamente distintas al modelo presentado por el índice de XPM. Éste representa la modulación de la fase de la señal de prueba, la que resultó ser inversamente proporcional a la frecuencia de modulación. A diferencia de él, en este otro modelo se ha considerado la conversión de las fluctuaciones de fase a intensidad a través de la dispersión de la fibra, cuya expresión está dada por la ecuación (26) y se observa que su eficiencia es proporcional a $\operatorname{Sin}\left(\beta_{2} \Omega^{2} L / 2\right)$. Así cuando aumentamos la frecuencia de modulación de la señal de bombeo la respuesta en frecuencia de XPM también aumentará.

\section{CONCLUSIONES}

El primer modelo nos muestra que, mientras el pulso se propaga a través de la fibra, la fase es modulada debido a la dependencia de intensidad del índice de refracción. Esta modulación de fase posee dos términos que se deben a SPM y a XPM, respectivamente. Ante la presencia de la dispersión, diferentes componentes espectrales de un pulso viajan con velocidades de grupo ligeramente distintas y que en conjunto con SPM y XPM, cambian la forma del pulso y su espectro. 
Del segundo modelo se concluye que XPM depende fuertemente de la separación entre los canales de prueba y de bombeo, así como también de la frecuencia de modulación de esta última. A medida que se aumenta el espaciamiento entre canales, el índice de XPM se hace más débil para una determinada frecuencia de modulación. Esto, porque el walk-off es mayor cuando los canales están más cercanos, provocando que el tiempo de interacción entre las señales de ambos canales sea mayor. A bajas frecuencias de modulación, el índice de XPM es mayor que a altas frecuencias, porque a bajas frecuencias, la duración del walk-off es mucho menor que el período de la señal de bombeo $T_{0}$ y la luz de prueba que se propaga experimenta un cambio de fase mayor que cuando el período de la señal de bombeo $T_{0}$ es menor que $T_{w}$.

El tercer modelo concluye que la respuesta de frecuencia de la diafonía inducida por XPM depende directamente de la potencia de la señal de bombeo y del espaciamiento entre los canales. Este modelo muestra características cualitativamente distintas al modelo anterior. A diferencia de él, aquí se considera la conversión de las fluctuaciones de fase a intensidad, por causa de la dispersión de la fibra.

\section{AGRADECIMIENTOS}

Este trabajo fue realizado en el marco del proyecto FONDEF DOOI1026, "Redes Ópticas para Internet del futuro".

\section{REFERENCIAS}

[1] M. Shtaif y M. Eistelt. "Analysis of Intensity Caused by Cross-Phase Modulation in Dispersive Optical Fibers". IEEE Photon. Technol. Lett. Vol. 10. $\mathrm{N}^{\mathrm{o}} 7$, pp. 979-981. July 1998.

[2] S. Betti y M.Giaconi. "Effect of the Cross-Phase Modulation on WDM Optical Systems: Analysis of Fiber Propagation". IEEE Photon. Technol. Lett. Vol. 13. No 4, pp. 305-307. April 2001.

[3] D. Breuer, K. Obermann y K. Petermann. "Comparison of $\mathrm{Nx} 40 \mathrm{Gbps}$ and $4 \mathrm{Nx} 10 \mathrm{Gbps}$ WDM Transmission Over Standard Single-Mode
Fiber at $1.55 \mathrm{~mm}$ ". IEEE Photon. Technol. Lett.. Vol. 10. $\mathrm{N}^{\circ} 12$, pp. 1793-1795. December 1998.

[4] D. Marcuse, A.R. Chraplyvy y W. Tkach. "Effect of fiber nonlinearity on long distance transmission". J. Lightwave Technol. Vol. 9, pp. 121-128. January 1991.

[5] T.K. Chiang, N. Kagi, T.K. Fong, M.E. Marhic y L.G. Kazovsky. "Cross-Phase Modulation in Dispersive fibers: Theorical and Experimental Investigation of the impact of Modulation Frequency ". IEE Photonics Technology Letters. Vol. 6, pp. 733-736. June 1994.

[6] T.K. Chiang, N. Kagi, M.E. Marhic y L.G. Kazovsky. "Cross-Phase Modulation in Fiber Links with Multiple Optical Amplifiers and Dispersion Compensators". J. Lightwave Technol. Vol.14, pp. 249-260. March 1996.

[7] R. Hui, K.R. Demarest y C.T. Allen. "Cross-Phase Modulation in Multispan WDM Optical Fiber Systems". J. Lightwave Technol. Vol. 17, pp. 10181026. Junio 1999.

[8] G.P. Agrawal, P.L. Baldeck y R.R. Alfano. "Temporal and spectral effects of cross-phase modulation on copropagating ultrashort pulses in optical fibers". Physical Review A. Vol. 40, pp. 5063-5072. November 1989.

[9] G.P. Agrawal. "Fiber Optics Communications Systems". John Wiley \& Sons, Inc. 3rd edition. New York, Estados Unidos. 2002.

[10] G.P. Agrawal. "Aplication of Nonlinear Fiber Optics”. Elsevier Sience, $1^{\text {st }}$ edition. California, United States. 2001.

[11] L.O. Jaraquemada. "Dispersión y Automodulación de Fase en Fibra Óptica Monomodo en Régimen no Lineal". Tesis para optar al grado de Magíster, Departamento Ingeniería Eléctrica, Universidad de Santiago de Chile. 2000.

[12] Especificaciones Corning SMF-28 Single Mode Optical Fiber. March 2002. Dirección web: http:// doclib.corning.com/OpticalFiber/pdf/pi1344.pdf. 